\title{
DebrisInterMixing-2.3: a finite volume solver for three-dimensional debris-flow simulations with two calibration parameters - Part 1: Model description
}

\author{
Albrecht von Boetticher ${ }^{1,3}$, Jens M. Turowski ${ }^{2}$, Brian W. McArdell ${ }^{3}$, Dieter Rickenmann ${ }^{3}$, and James W. Kirchner Jan $^{1,3}$ \\ ${ }^{1}$ Department of Environmental Systems Science, ETH Zentrum, CHN H41, 8092 Zürich, Switzerland \\ ${ }^{2}$ Helmholtz-Centre Potsdam GFZ German Research Center for Geosciences, Telegrafenberg, 14473 Potsdam, Germany \\ ${ }^{3}$ Swiss Federal Research Institute WSL, Zürcherstrasse 111, 8903 Birmensdorf, Switzerland
}

Correspondence to: Albrecht von Boetticher (albrecht.vonboetticher@usys.ethz.ch)

Received: 26 June 2015 - Published in Geosci. Model Dev. Discuss.: 13 August 2015

Revised: 15 July 2016 - Accepted: 18 July 2016 - Published: 31 August 2016

\begin{abstract}
Here, we present a three-dimensional fluid dynamic solver that simulates debris flows as a mixture of two fluids (a Coulomb viscoplastic model of the gravel mixed with a Herschel-Bulkley representation of the fine material suspension) in combination with an additional unmixed phase representing the air and the free surface. We link all rheological parameters to the material composition, i.e., to water content, clay content, and mineral composition, content of sand and gravel, and the gravel's friction angle; the user must specify only two free model parameters. The volume-of-fluid ( $\mathrm{VoF})$ approach is used to combine the mixed phase and the air phase into a single cellaveraged Navier-Stokes equation for incompressible flow, based on code adapted from standard solvers of the opensource CFD software OpenFOAM. This effectively singlephase mixture VoF method saves computational costs compared to the more sophisticated drag-force-based multiphase models. Thus, complex three-dimensional flow structures can be simulated while accounting for the pressure- and shear-rate-dependent rheology.
\end{abstract}

\section{Introduction}

Debris flows typically occur in steep mountain channels. They are characterized by unsteady flows of water together with different contents of clay, silt, sand, gravel, and larger particles, resulting in a dense and often rapidly moving mixture mass. They are often triggered by heavy rainfall and can cause massive damage (Petley et al., 2007; Hilker et al., 2009). Their importance has increased due to extensive settlement in mountainous regions and also due to their sensitivity to climate change (Guthrie et al., 2010). Their damage potential is not limited to direct impact; severe damage can also be caused by debris flows blocking channels and thus inducing overtopping of the banks by subsequent flows (Tang et al., 2011).

Modeling debris flows is a central part of debris-flow research, because measuring the detailed processes in debrisflow experiments or in the field is challenging. It is still uncertain how laboratory tests can be scaled to represent real flow events, and the inhomogeneous mixture of gravel and fine material brings about interactions of granular flow and viscous forces such as drag and pore pressure that are difficult to track with the present measurement techniques at reasonable cost. As a consequence, the rheological behavior of debris-flow material remains incompletely understood.

Typically, current numerical modeling approaches cannot predict run-out distances or impact pressures of debris flows without parameter calibration that is based on simulations of previous well-documented events that occurred at the same site. This clearly represents a challenge in practical applications, because reliable calibration data are rarely available. Due to the complex physics of debris flows, real flows can only be accurately described by dynamical models that include strong phase interactions between granular and viscous fluid phases with several physical parameters (Pudasaini, 2012). From a practical application point of view and 
guided by considerations of computational efficiency, here we neglect such phase interactions and restrict ourselves to an effectively single-phase mixture flow simulation. All currently applied debris-flow models that use a two-phase description of the debris-flow material are depth averaged or 2D. Three-dimensional debris-flow models with momentum exchange between phases have, up to now, been limited to academic cases due to their high numerical costs. Depthaveraged approximations are most applicable to flows over smooth basal surfaces with gradual changes in slope. These approximations are less valid when topography changes are abrupt, such as close to flow obstacles. They are also less applicable when the flow exhibits strong gradients in accelerations, such as during flow initiation and deposition, or in strongly converging and diverging flows. For such cases, we need a physically complete description of the flow dynamics without depth averaging (Domnik and Pudasaini, 2012). The essential physics of debris flows can be better retained by developing a full-dimensional flow model and then directly solving the model equations without reducing their dimension. The currently available models also contain many parameters that must be estimated based on measurements, or fitted to site-specific field data, limiting their applicability to real-world problems.

Here, we provide a greatly simplified but effective solution linking the rheological model of the debris-flow material to field values such as grain size distribution and water content. The approach aims to link the knowledge of field experts for estimating the release volume and material composition with recent advances that account for complex flow phenomena, by using three-dimensional computational fluid dynamics with reasonable computational costs. The parameters of the two resulting rheology models for the two mixing fluids are linked to material properties such that the model setup can be based on material samples collected from the field, yielding a model that has two free parameters for calibration. One mixture component represents the suspension of finer particles with water (also simply called slurry in this paper) and a second component accounts for the pressuredependent flow behavior of gravel. The two components result in a debris bulk mixture with contributions of the two different rheology models, weighted by the corresponding component concentrations. A third gas component is kept unmixed to model the free surface. The focus is on the flow and deposition process and the release body needs to be user defined.

\section{Modeling approach}

The debris-flow material can be considered as a combination of a granular component and an interstitial fluid composed of a fine material suspension. The interstitial fluid was successfully modeled in the past as a shear-rate-dependent Herschel-Bulkley fluid (Coussot et al., 1998). Because pres- sure and shear drive the energy dissipation of particleto-particle contacts, the shear rate substantially influences the energy dissipation within the granular phase. While the two-phase models of Iverson and Denlinger (2001) and Pitman and Le (2005) treated the granular phase as a shearrate-independent Mohr-Coulomb plastic material, dry granular material has been successfully modeled as a viscoplastic fluid by Ancey (2007), Forterre and Pouliquen (2008), Balmforth and Frigaard (2007), and Jop et al. (2006). We follow the suggestions given by Pudasaini (2012) to account for the non-Newtonian behavior of the fluid and the shearand pressure-dependent Coulomb viscoplastic behavior of the granular phase, as proposed by Domnik et al. (2013). Several modeling approaches to account for the two-phase nature of debris flows used depth-averaged mass and momentum balance equations for each phase coupled by drag models (e.g., Bozhinskiy and Nazarov, 2000, Pitman and Le, 2005 and Bouchut et al., 2015). Pudasaini (2012) proposed a more comprehensive two-phase mass-flow model that includes general drag, buoyancy, virtual mass, and enhanced nonNewtonian viscous stress in which the solid volume fraction evolves dynamically. We apply a numerically more efficient method and treat the debris-flow material as one mixture (Iverson and Denlinger, 2001, Pudasaini et al., 2005a), with phase-averaged properties described by a single set of Navier-Stokes-type equations. The resulting reduction in numerical costs allows us to model the three-dimensional momentum transfer in the fluid as well as the free-surface flow over complex terrain and obstacles.

We assume that the velocity of the gravel is the same as the velocity of the fluid. This assumption is motivated from application and would not be valid for general debris flows with interstitial fluid of low viscosity (i.e., a slurry with low concentration of fine material and large water content). The assumption of equal velocity of gravel and interstitial fluid in one cell leads to a constant composition of the mixture by means of phase concentrations over the entire flow process. This basic model can be seen as a counterpart to the mixture model of Iverson and Denlinger (2001), extended by resolving the three-dimensional flow structure in combination with a pressure- and shear-rate-dependent rheology linked to the material composition. The basic model presented here focuses on the role of pressure- and shear-rate-dependent flow behavior of the gravel, in combination with the shear-ratedependent rheology of the slurry.

We base our model concept on the well-established finite volume solver interFoam, which is designed for incompressible two-phase flow simulations of immiscible fluids (Deshpande et al., 2012). A standard extension named interMixingFoam introduces two mixing phases without momentum exchange, coupled to a third unmixed phase by surface tension. The present method limits the physics of flow in terms of the interactions between phases such as drag, buoyancy, virtual mass, non-Newtonian viscous stress, and evolving volume fraction of the solid phase (Pitman and Le, 2005; 
Table 1. Nomenclature.

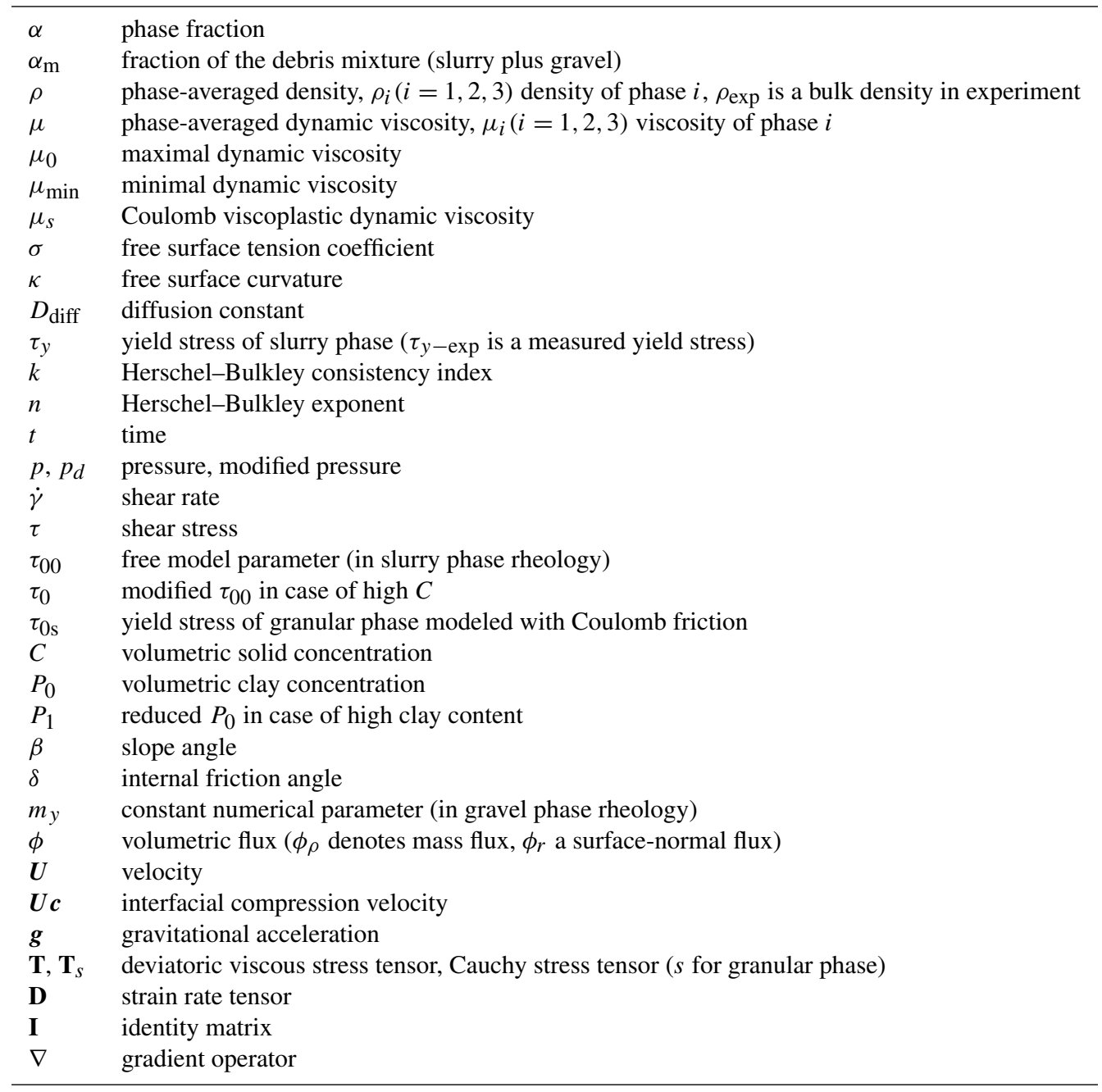

Pudasaini, 2012). Numerical costs are kept reasonable due to the volume-of-fluid (VoF) method (Hirt and Nichols, 1981), which solves only one Navier-Stokes equation system for all phases. The viscosity and density of each grid cell is calculated as a concentration-weighted average of the viscosities and densities of the phases that are present in the cell. Between the two mixing phases of gravel and slurry, the interaction reduces to this averaging of density and viscosity. In this way, the coupling between driving forces, topography and three-dimensional flow-dependent internal friction can be addressed for each phase separately, accounting for the fundamental differences in flow mechanisms of granular and viscoplastic fluid flow that arise from the presence or absence of Coulomb friction (Fig. 1). We apply linear concentrationweighted averaging of viscosities for estimating the bulk viscosity of a mixture for simplicity. Nevertheless, interacting forces such as drag between the grains and the fluid do not appear in our model because we set solid velocity equal to fluid velocity, and thus, from the physical point of view, this is a debris bulk model.

\subsection{Governing equations}

Assuming isothermal incompressible phases without mass transfer, we separate the modeled space into a gas region denoting the air and a region of two mixed liquid components. The VoF method used here determines the volume fractions of all components in an arbitrary control volume by using an indicator function which yields a fraction field for each component. The fraction field represents the probability that a component is present at a certain point in space and time (Hill, 1998). The air fraction may be defined in relation to the fraction of the mixed fluid $\alpha_{\mathrm{m}}$ as

$\alpha_{1}=1-\alpha_{\mathrm{m}}$, 

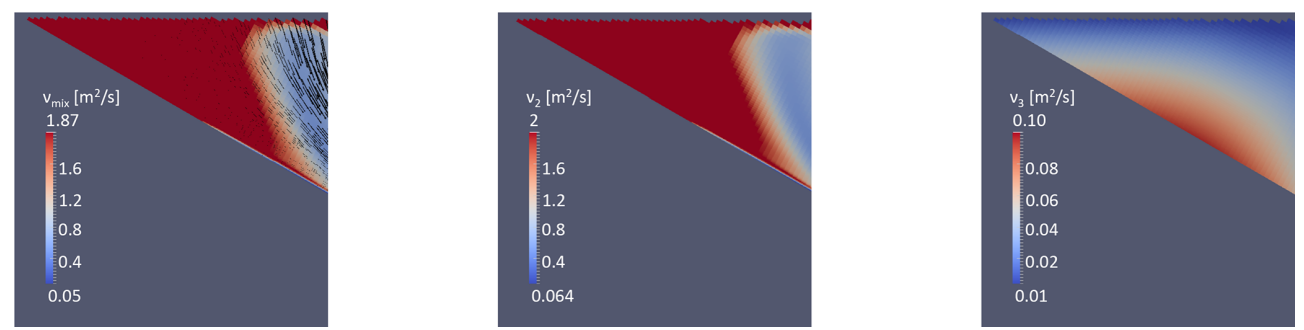

Figure 1. Viscosity distribution (indicated by color scale) along a $28 \mathrm{~cm}$ long section through the modeled $0.01 \mathrm{~m}^{3}$ release block $0.2 \mathrm{~s}$ after release, corresponding to the experimental setup of Hürlimann et al. (2015). The starting motion (black velocity arrows) with corresponding viscosity distribution of the mixture (left) is a consequence of blending pure shear-rate-dependent slurry-phase rheology (center) with the pressure- and shear-rate-dependent gravel phase rheology that accounts for Coulomb friction (right). Because the gravel concentration in this example is low, its effect on the overall viscosity is small.

and the mixed fluid $\alpha_{\mathrm{m}}$ may be defined as the sum of the constant fractions of the mixing phases $\alpha_{2}$ and $\alpha_{3}$ :

$\alpha_{\mathrm{m}}=\alpha_{2}+\alpha_{3}$.

The debris bulk motion is defined by the continuity equation together with the transport and momentum equations:

$\nabla \cdot \boldsymbol{U}=0$,
$\frac{\partial \alpha_{\mathrm{m}}}{\partial t}+\nabla \cdot\left(\boldsymbol{U} \alpha_{\mathrm{m}}\right)=0$,

and

$\frac{\partial(\rho \boldsymbol{U})}{\partial t}+\nabla \cdot(\rho \boldsymbol{U} \times \boldsymbol{U})=-\nabla p+\nabla \cdot \mathbf{T}+\rho \boldsymbol{f}$,

where $t$ denotes time and $\boldsymbol{U}$ represents the debris bulk velocity field, $\mathbf{T}$ is the deviatoric viscous stress tensor for the mixture, $\rho$ is the phase-averaged bulk density, $p$ denotes pressure, and $\boldsymbol{f}$ stands for body forces per unit mass. We assume incompressible material and all fractions are convected with the same bulk velocity. Therefore, differences in phase velocities, and thus the interaction forces, such as drag between the grains and the fluid, are neglected. An efficient technique of the VoF method convects the fraction field $\alpha_{\mathrm{m}}$ as an invariant with the divergence-free flow field $\boldsymbol{U}$ that is known from previous time steps:

$\frac{\partial \alpha_{\mathrm{m}}}{\partial t}+\nabla \cdot\left(\boldsymbol{U} \alpha_{\mathrm{m}}\right)+\nabla \cdot\left(\alpha_{1} \boldsymbol{U}_{\mathrm{c}}\right)=0$,

where $\boldsymbol{U}_{\mathrm{c}}$ is an artificial interfacial compression velocity acting perpendicular to the interface between the gas region and the mixed liquid phases. The method allows a reconstruction of the free surface with high accuracy if the grid resolution is sufficient (Berberović et al., 2009; Hoang et al., 2012; Deshpande et al., 2012; Hänsch et al., 2013). The details about the interface compression technique, the related discretization, and numerical schemes to solve Eq. (6) are given in Deshpande et al. (2012). However, to allow diffusion between the mixing constituents of the slurry $\alpha_{2}$ and the gravel $\alpha_{3}$ in case of initially unequally distributed concentrations, our modified version of the interMixingFoam solver applies Eq. (6) separately to each mixing component including diffusion:

$\frac{\partial \alpha_{i}}{\partial t}+\nabla \cdot\left(\boldsymbol{U} \alpha_{i}\right)-D_{\mathrm{diff}} \nabla^{2} \alpha_{i}+\nabla \cdot\left(\alpha_{1} \boldsymbol{U}_{\mathrm{c}}\right)=0$,

where $i=2,3$ denote the slurry and gravel constituents and $D_{\text {diff }}$ is the diffusion constant that is set to a negligible small value within the scope of this paper.

The discrete form of Eq. (7) is derived by integrating over the volume $V$ of a finite cell of a grid discretization of the simulated space, which is done in the finite volume method by applying the Gauss theorem over the cell faces. The advective phase fluxes $\phi_{1 \ldots 3}$ are obtained by interpolating the cell values of $\alpha_{1}, \alpha_{2}$, and $\alpha_{3}$ to the cell surfaces and by multiplying them with the flux $\phi$ through the surface, which is known from the current velocity field. To keep the air phase unmixed, it is necessary to determine the flux $\phi_{r}$ through the interface between air and the debris flow mixture, and to subtract it from the calculated phase fluxes $\phi_{1 \ldots 3}$. Inherited from the original interMixingFoam solver (OpenFOAMFoundation, 2016a), limiters are applied during this step to bound the fluxes to keep phase concentrations between 0 and 1 . With known fluxes $\phi_{1 \ldots 3}$, the scalar transport equation for each phase takes the form

$\frac{\partial}{\partial t} \alpha_{i}+\nabla\left(\phi_{i}\right)-D_{\text {diff }} \nabla^{2} \alpha_{i}=0$.

Equation (8) is the implemented scalar transport equation solved for each constituent using first-order Euler schemes for the time derivative terms, as has been recommended for liquid column breakout simulations (Hänsch et al., 2013).

After solving the scalar transport equations, the complete mass flux $\phi_{\rho}$ is constructed from the updated volumetric fraction concentrations:

$\phi_{\rho}=\phi_{1} \rho_{1}+\phi_{2} \rho_{2}+\phi_{3} \rho_{3}$, 


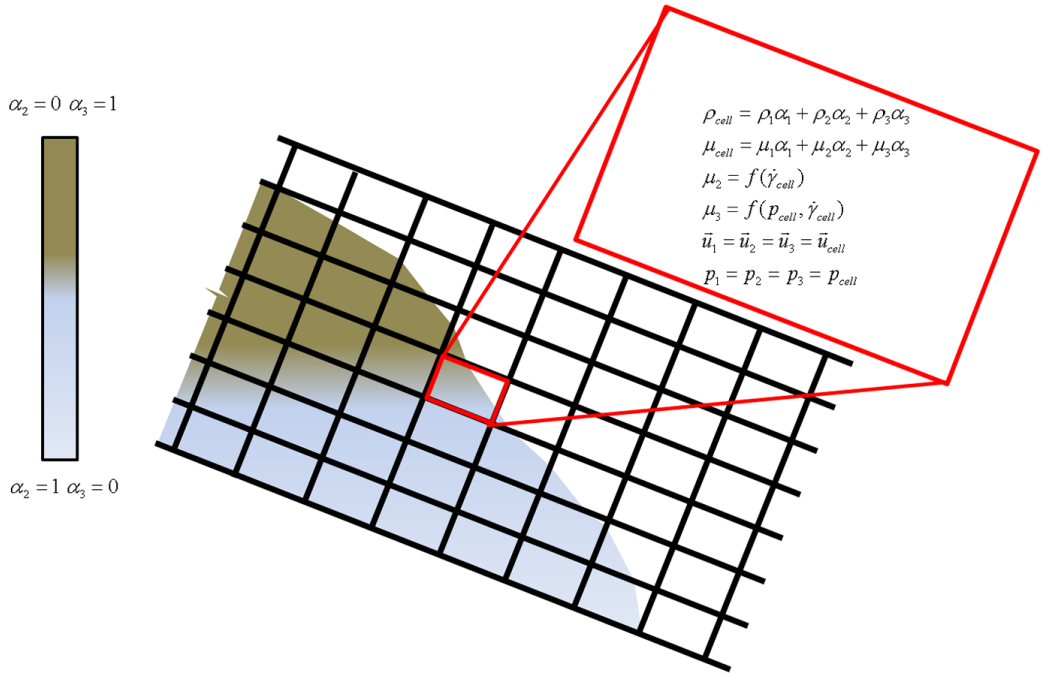

Figure 2. Longitudinal section through a debris-flow front discretized with finite-volume cells, showing the constitutive equations for one cell with density $\rho$ and viscosity $\mu$, given the densities $\rho_{1 \ldots 3}$, viscosities $\mu_{1 \ldots 3}$, and proportions $\alpha_{1 \ldots 3}$ of phases present. The numbers 1 , 2 , and 3 denote the air (white colored cell content), mud, and gravel phases, respectively.

where $\rho_{1 \ldots 3}$ denote the constant densities of the corresponding phases and $\phi_{1 \ldots 3}$ are the corresponding fluxes. The complete mass flux $\phi_{\rho}$ is used in the implementation to describe the second term of the momentum equation (Eq. 14) as described in Appendix A. Figure 2 illustrates how the phase volume distributions $\alpha_{1}$ (air), $\alpha_{2}$ (slurry), and $\alpha_{3}$ (gravel) are used to derive cell-averaged properties of the continuum.

The conservation of mass and momentum is averaged with respect to the phase fraction $\alpha$ of each phase. The density field is defined as

$\rho=\sum_{i} \rho_{i} \alpha_{i}$

where $\rho_{i}$ denotes density of phase $i$ and the phase density is assumed to be constant.

The deviatoric viscous stress tensor $\mathbf{T}$ is defined based on the mean strain rate tensor $\mathbf{D}$ that denotes the symmetric part of the velocity gradient tensor derived from the phaseaveraged flow field:

$\mathbf{D}=\frac{1}{2}\left[\nabla \boldsymbol{U}+(\nabla \boldsymbol{U})^{T}\right]$,

and

$\mathbf{T}=2 \mu \mathbf{D}$.

Equation (12) was derived accounting for Eq. (3) and $\mu$ is the phase-averaged dynamic viscosity, which is simplified in analogy to Eq. (10) as the concentration-weighted average of the corresponding phase viscosities:

$\mu=\sum_{i} \mu_{i} \alpha_{i}$.
With the continuity Eq. (3), the term $\nabla \cdot \mathbf{T}$ in Eq. (5) is written as $\nabla \cdot(\mu \nabla \boldsymbol{U})+(\nabla \boldsymbol{U}) \cdot \nabla \mu$ to ease discretization. The body forces $f$ in the momentum equation account for gravity and for the effects of surface tension. The surface tension at the interface between the debris-flow mixture and air is modeled as a force per unit volume by applying a surface tension coefficient $\sigma$. Although the surface tension can be considered to have a minor influence on debris-flow behavior, it allows an adequate reproduction of surface-flow patterns observed in laboratory-scale experiments used for validation (von Boetticher et al., 2015). The momentum conservation including gravitational acceleration $\boldsymbol{g}$ and surface tension is defined in our model as

$$
\begin{aligned}
& \frac{\partial(\rho \boldsymbol{U})}{\partial t}+\nabla \cdot(\rho \boldsymbol{U} \times \boldsymbol{U})=-\nabla p_{d}+\nabla \cdot(\mu \nabla \boldsymbol{U}) \\
& \quad+(\nabla \boldsymbol{U}) \cdot \nabla \mu-\boldsymbol{g} \cdot \boldsymbol{x} \nabla \rho+\sigma \kappa \nabla \alpha_{1},
\end{aligned}
$$

where $\kappa$ denotes the local interfacial curvature and $\boldsymbol{x}$ stands for position. The modified pressure $p_{d}$ is employed in the solver to overcome some difficulties with boundary conditions in flow simulations with density gradients. In case the free surface lies within an inclined wall forming a no-slip boundary condition, the normal component of the pressure gradient must be different for the gas phase and the mixture due to the hydrostatic component $\rho g$. It is common to introduce a modified pressure $p_{d}$ related to the pressure $p$ by

$p_{d}=p-\rho \boldsymbol{g} \cdot \boldsymbol{x}$.

The gradient of the modified pressure includes the static pressure gradient and contributions that arise from the density 
gradient as well as a body force due to gravity (Berberović et al., 2009).

Together with the continuity Eq. (3), Eq. (14) allows us to calculate the pressure- and gravity-driven velocities. The corresponding discretization and solution procedure with the PISO (pressure-implicit with splitting of operators; Issa, 1986) algorithm is provided in Appendix A. The set of equations governing the flow in our model are Eqs. (8)-(15) together with the continuity Eq. (3). In the following section, we present the rheology models that define the viscosity components for Eq. (13).

\subsection{Rheology model for the fine sediment suspension}

The viscosity of the gas phase, $\mu_{1}$ is chosen constant. The introduction of two mixing phases is necessary to distinguish between the pressure-dependent flow behavior of gravel and the shear-thinning viscosity of the suspension of finer particles with water. The rheology of mixtures of water with clay and sand can be described by the Herschel-Bulkley rheology law (Coussot et al., 1998), which defines the shear stress in the fluid as

$\tau=\tau_{y}+k \dot{\gamma}^{n}$,

where $\tau_{y}$ is a yield stress below which the fluid acts like a solid, $k$ is a consistency index for the viscosity of the sheared material, $\dot{\gamma}$ is the shear rate, and $n$ defines the shearthinning $(n<1)$ or shear-thickening $(n>1)$ behavior. In OpenFOAM, the shear rate is derived in 3-D from the strain rate tensor $\mathbf{D}$ :

$\dot{\gamma}=\sqrt{2 \mathbf{D}: \mathbf{D}}$.

The shear rate is based on the strain rate tensor to exclude the rotation velocity tensor that does not contribute to the deformation of the fluid body. The model can be rewritten as a generalized Newtonian fluid model to define the shear-ratedependent effective kinematic viscosity of the slurry phase as

$\mu_{2}=k|\dot{\gamma}|^{n-1}+\tau_{y}|\dot{\gamma}|^{-1}$

if the viscosity is below an upper limit $\mu_{0}$ and

$\mu_{2}=\mu_{0}$

if the viscosity is higher, to ensure numerical stability.

With $n=1$, the model simplifies to the Bingham rheology model that has been widely used to describe debris-flow behavior in the past (Ancey, 2007). It may be reasonable to imagine the rheology parameters to be dependent on the state of the flow. However, even with the implicit assumption that the coefficients are a property of the material and not of the state of the flow, the Herschel-Bulkley rheology law has been rarely applied in debris-flow modeling due to three rheology parameters. We avoid this problem by assuming some rheology parameters to be defined by measurable material properties as described below.

\section{Determination of rheology model parameters based on material properties}

Results from recent publications allow the reduction of the number of free Herschel-Bulkley parameters to two. If the coarser grain fraction is assumed to be in the gravel phase, the Herschel-Bulkley parameters for the finer material can be linked to material properties that can be measured using simple standard geotechnical tests. According to Coussot et al. (1998), the exponent $n$ can be assumed constant at $1 / 3$, and $k$ can be roughly estimated as $b \tau_{y}$, with the constant $b=0.3 \mathrm{~s}^{-\mathrm{n}}$ for mixtures with maximum grainsizes $<0.4 \mathrm{~mm}$ (Coussot et al., 1998). An approach for estimating the yield stress $\tau_{y}$ based on water content, clay fraction and composition, and the solid concentration of the entire debris-flow material was proposed by Yu et al. (2013) as

$\tau_{y}=\tau_{0} C^{2} e^{22\left(C P_{1}\right)}$,

where $C$ (a constant) is the volumetric solid concentration of the mixture (the volume of all solid particles including fine material relative to the volume of the debris-flow material including water), $P_{1}=0.7 P_{0}$ when $P_{0}>0.27$, and $P_{1}=P_{0}$ if $P_{0}<=0.27$, and

$$
\begin{aligned}
P_{0} & =C_{\text {kaolinite }+ \text { chlorite }}+1.3 C_{\text {illite }} \\
& +1.7 C_{\text {montmorillonite }},
\end{aligned}
$$

where the subscript of $C$ refers to the volumetric concentration (relative to the total volume of all solid particles and water) of the corresponding mineral. The discontinuity of $P_{1}$ at a modified clay concentration of $P_{0}=0.27$ is a coarse adjustment to a more-or-less sudden change observed in the experimental behavior.

For $C<0.47, \tau_{0}$ is equal to $\tau_{00}$ and otherwise $\tau_{0}$ can be calculated by

$\tau_{0}=\tau_{00} e^{5(C-0.47)}$,

where $\tau_{00}$ is the remaining free parameter which we use to account for the grid-size dependency of the shear rate (Yu et al., 2013). The change in the definition of $\tau_{0}$ at solid concentrations exceeding 0.47 accounts for a threshold between weak coherent and medium coherent debris-flow material.

Yu et al. (2013) compared this method of estimating the yield stress $\tau_{y}$ to experimental results they obtained from a set of 514 flume experiments with mixtures of water and clay with fine and coarse sand and less than $5 \%$ gravel. They determined the yield stress by releasing the material mixture 
from a reservoir into an inclined channel of $0.2 \mathrm{~m}$ width and by increasing the inclination slightly until remobilization occurred after the material came to rest. The experimental yield stress $\tau_{y-\exp }$ was then simplified as

$\tau_{y-\exp }=\rho_{\exp } g h \sin (\beta)$,

where $\rho_{\text {exp }}$ is the density of the applied mixture, $h$ is the maximum accumulation thickness of the deposit, and $\beta$ is the slope inclination. In addition, they compared the calculated yield stress of Eq. (20) with experimental yield stresses reported by a number of authors including Coussot et al. (1998) and Ancey and Jorrot (2001). Ancey and Jorrot (2001) used 2 and $3 \mathrm{~mm}$ glass beads in a kaolinite dispersion as well as fine sand-kaolinite-water mixtures. Up to yield stresses of about $200 \mathrm{~Pa}$ the yield stresses estimated by Eq. (20) fit the observed ones well. Thus, the yield stresses of sand-clay mixtures with water can be estimated using Eq. (20) based on the volumetric concentration of the debris in the water-solids mixture and based on the percentages of different clays in the fraction of fine material. Adjustments to the numbers for calculating $P_{0}$ may be necessary to account for the activity of other clays.

The remaining uncertainties concern the assumptions about the value of $n$, and that $k$ can be defined in such simple dependency to $\tau_{y}$ in the presence of coarser sand. Experiments seem to confirm that $n$ increases in presence of coarser material (Imran et al., 2001), but further research is needed to quantify this effect. Remaitre et al. (2005) found $n$ to vary from 0.27 to 0.36 . Schatzmann et al. (2003) used $n=0.33$ to reproduce measured curves obtained with a mixture of 27.5 volumetric percent slurry with $30 \%$ gravel where gravel grain sizes ranged from 3 to $10 \mathrm{~mm}$. He applied $n=0.5$ to fit the Herschel-Bulkley model to the experiment with $22.5 \%$ slurry and $30 \%$ gravel. Based on the laboratory-scale experiments that are presented in von Boetticher et al. (2015), we have chosen $n=0.34$ to obtain the best fit for the simulation of large-scale debris-flow experiments. However, other debris-flow mixtures may demand a recalibration of $n$ because natural debris-flow mixtures cover a wide spectrum from shear thinning to shear thickening behavior. Thus, we consider $n$ as the second model calibration parameter. The model calibration is constricted to $\tau_{00}$, which accounts for the grid resolution sensitivity of the shear rate and $n$, which allows adjusting the model to shear thinning or shear thickening mixtures.

\subsection{Representation of gravel by a Coulomb viscoplastic rheology}

With a novel model, Domnik and Pudasaini (2012) showed that introducing a Coulomb viscoplastic sliding law to replace a simple no-slip boundary condition completely changes the flow dynamics and the depth variations in velocity and dynamic pressure. They show that with a Coulomb viscoplastic sliding law, the observable shearing mainly occurs close to the sliding surface, in agreement with observations but in contrast to the no-slip boundary condition. In our model, during acceleration and high-speed flow, the shearthinning behavior of both the fluid and the granular phase dominate the viscosity. However, the representation of gravel by a pressure and rate-dependent Coulomb viscoplastic rheology becomes important as soon as the material experiences high pressures, accompanied by reduction in shear due to decelerations. This may be caused by channel slope reductions, diverging or converging flows, and interactions with obstacles (see, e.g., Pudasaini et al., 2005b; Domnik et al., 2013). Flows of granular material could be modeled as viscoplastic fluids (Ancey, 2007; Forterre and Pouliquen, 2008; Balmforth and Frigaard, 2007; Jop et al., 2006) as cited by Domnik and Pudasaini (2012). Based on Ishii (1975), the granular Cauchy stress tensor $\mathbf{T}_{s}$ can be written as

$\mathbf{T}_{s}=-p \mathbf{I}+2 \mu_{s} \mathbf{D}$,

where $p \mathbf{I}$ is the pressure times the identity matrix and $\mu_{s}$ is the corresponding dynamic viscosity, which was modeled by Domnik and Pudasaini (2012) as

$\mu_{s}=\mu_{\min }+\frac{\tau_{0 \mathrm{~s}}}{\|\mathbf{D}\|}\left[1-e^{-m_{y}\|\mathbf{D}\|}\right]$,

where $\mu_{\min }$ is a minimal dynamic viscosity, $\tau_{0 \mathrm{~s}}$ is a yield stress, and $\|\mathbf{D}\|$ is the norm of the strain-rate tensor defined by the authors as

$\|\mathbf{D}\|=\sqrt{2 \operatorname{tr}\left(\mathbf{D}^{2}\right)}$.

In Eq. (25), $m_{y}$ is a numerical parameter with units of seconds which we will keep constant. Domnik et al. (2013) derived the yield stress as a pressure-dependent Coulomb friction, $p \cdot \sin (\delta)$ where $\delta$ is the internal friction angle:

$\mu_{3}=\mu_{\min }+\frac{p \cdot \sin (\delta)}{\|\mathbf{D}\|}\left[1-e^{-m_{y}\|\mathbf{D}\|}\right]$.

Here, this Coulomb viscoplastic rheology model is used to describe the gravel phase. The pressure- and shear-dependent viscosity is calculated in every cell with the corresponding local pressure $p$ and strain-rate tensor $\mathbf{D}$ derived from the phase-averaged flow field.

\section{Gravel phase properties}

The Coulomb viscoplastic rheology law developed by Domnik et al. (2013) includes two parameters: one material parameter that can be measured (the friction angle $\delta$ ) and a numerical parameter $m_{y}$ introduced for numerical reasons to influence the smooth transition between yielded and unyielded 


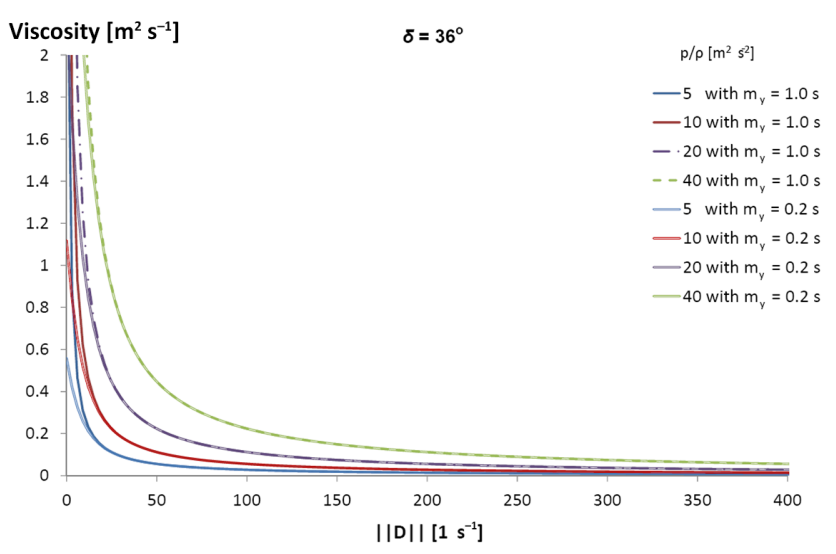

Figure 3. Dependency of the kinematic gravel phase viscosity $v_{S}$ (normalized by density) on the norm of the strain rate tensor $\|D\|$ at different levels of pressure normalized by density for $m_{y}=1 \mathrm{~s}$, $m_{y}=0.2 \mathrm{~s}$, and a friction angle $\delta=36^{\circ}$.

regions. For smaller values of $m_{y}$, the transition is smoother. In the absence of shear, to achieve a viscosity representing a Coulomb friction equal to $p \cdot \sin (\delta)$ where $p$ is the local pressure, $m_{y}$ needs to be equal to $1 \mathrm{~s}$. However, the development of $\mu_{s}$ under large pressure or strong shear is the same for both $m_{y}=1 \mathrm{~s}$ and $m_{y}=0.2 \mathrm{~s}$. So, we choose $m_{y}$ to be constant and equal to $0.2 \mathrm{~s}$ for all simulations. However, we mention that parts of the nearly immobile material that experience little pressure (in general, immobile material close to the surface) show a significant reduction in viscosity when $m_{y}=0.2 \mathrm{~s}$ (Fig. 3). As a consequence, $m_{y}$ minimally affects debris-flow release and flow at large scales, but slowly moving material with a shallow flow depth in a run-out plane may develop front fingering (which is dependent on and sensitive to the value of $m_{y}$ ) by allowing sudden local solidification.

For small friction angles, the modeled viscosity of the gravel phase decreases rapidly with increasing shear. Larger friction angles increase the viscosity and extend the pressure dependency to larger shear rates (Fig. 4). We estimated the friction angle $\delta$ based on the maximum angle of repose in tilt-table tests of the gravel. In our laboratory experiments, we determined the friction angle in a simple adaptation of the method of Deganutti et al. (2011) by tilting a large box with loose material until a second failure of the material body occurred.

In analogy to the Herschel-Bulkley implementation, an upper limit for the viscosity is implemented to maintain numerical stability. Pressure-dependent viscosity in the incompressible Navier-Stokes equations causes numerical instability as soon as the eigenvalues of the symmetric part of the local velocity gradient become larger than $1 /(2(\delta \mu / \delta p))$. Following Renardy (1986), we locally limit the viscosity to keep it below a corresponding local stability limit.

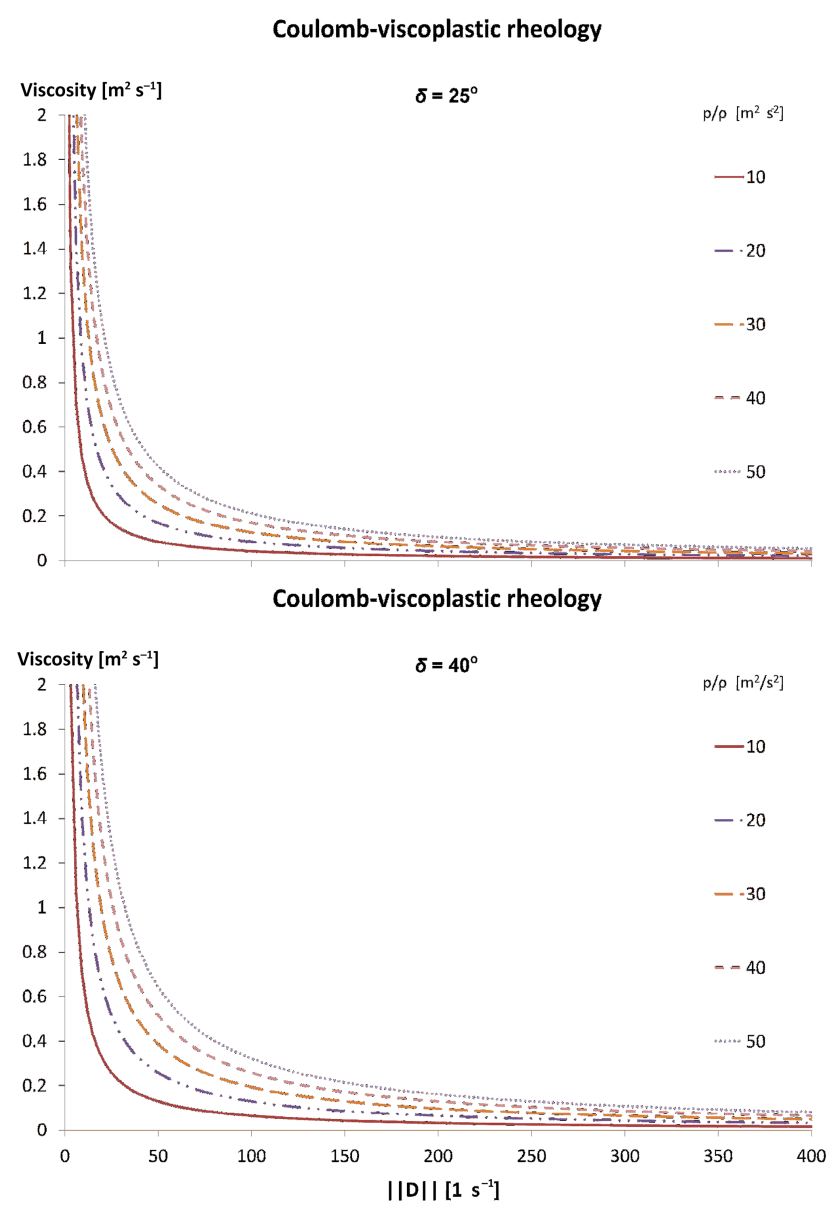

Figure 4. Dependency of the kinematic gravel phase viscosity (for friction angle $\delta=25^{\circ}$ and $40^{\circ}$ ) on the norm of the strain rate tensor $\|D\|$ at different levels of pressure normalized by density, for $m_{y}=$ $1 \mathrm{~s}$.

\section{Quality characteristics of the model}

\subsection{Modeled interaction between granular and fluid constituents}

As stated before, debris flows are multiphase flow processes where solid particles and interstitial fluid and probably gas phases interact. The model presented applies cell-averaged values of the mixtures and does not allow for consideration of interactions between a granular and a fluid phase. However, a dynamic evolution of the gravel and slurry concentration is the necessary next step. A two-way coupling to a Lagrangian particle simulation could deliver an alternative solution.

\subsection{Advantages of full-dimensional mass flow}

Laboratory and field observations show that the rapid flow regime is characterized by relatively uniform velocity profiles with depth, and dominant sliding at the base. By contrast, in the depositional regime and particularly during tran- 
sitions from rapid flow to the deposition regime, shocklike structures form and a predominantly basal sliding flow changes into a surface boundary layer flow, which, further downstream, quickly slows and eventually settles. This transition has been observed in the laboratory with granular PIV (particle image velocimetry) measurements for rapidly moving granular material impinging on a rigid wall perpendicular to the bed, leading to strong shearing through the flow depth (Pudasaini et al., 2007). The scaling of impact pressures on rigid obstacles with obstacle size is complex (Bugnion et al., 2012); the spatial distribution depends on the local flow structure (Scheidl et al., 2013) and the distribution of forces over the flow depth is non-trivial and highly dynamic (Berger et al., 2011). Thus, although the rapid flow regime can be reasonably approximated by a depthintegrated dynamical model, adequately simulating the deposition regime and interactions with obstacles requires resolving the three-dimensional flow structure. Our attempts to consider the three-dimensional flow structure of debris flows were motivated by the need to predict the dynamic loading of shallow landslide impacts to flexible protection barriers (von Boetticher, 2013) and the corresponding research in full-dimensional model development is a topic of ongoing interest (Leonardi et al., 2016). In the model presented here, we aim to account for both the three-dimensional flow structure and the corresponding influence of pressure. Domnik et al. (2013) developed a two-dimensional Coulomb viscoplastic model and applied it for inclined channel flows of granular materials from initiation to deposition. They proposed a pressure-dependent yield strength to account for the frictional nature of granular materials. The interaction of the flow with the solid boundary was modeled by a pressure- and rate-dependent Coulomb viscoplastic sliding law. In regions where depth-averaging becomes inaccurate, like in the initiation and deposition regions, and particularly in interactions with obstacles, three-dimensional models are essential because in these regions the momentum transfer must be considered in all directions. Thus, prediction of the velocity variations with depth, and the full dynamical and internal pressure, can only be obtained through three-dimensional modeling (Domnik et al., 2013). These dynamical quantities can be adequately described by a Coulomb viscoplastic material with a pressure-dependent yield stress expressed in terms of the internal friction angle, the only material parameter in their model. The pressure dependency introduces an additional need to account for the three-dimensional flow structure.

\subsection{Effects of time step size on rheology}

Brodani-Minussi and deFreitas Maciel (2012) simulated dam-break experiments of a Herschel-Bulkley fluid using the VoF approach, and compared the results with published shallow-water-equation-based models. Especially for the first instant after the material release, the application of shallow-water equations seems to introduce errors that are propagated throughout the process, leading to erroneous run-out estimates. A similar problem arises when modeling debris-flow impacts against obstacles. Simulating the impact of material with velocity-dependent rheology that is kept constant over the time step although it actually changes with the changing flow leads to an accumulating over- or underestimation of energy dissipation. For example, if a shearthinning material undergoes acceleration, and thus an increase in its shear rate, viscosity calculations based on its shear rate at the beginning of each time step would overestimate the average viscosity (and underestimate the change in shear rate) over the whole time step. As a consequence, the resulting viscosity at the end of the time step would be underestimated, which would amplify the overestimation of viscosity in the next time step. Conversely, at an impact, the sudden deceleration causes an underestimation of viscosity over the time step length, leading to an overestimated velocity that again amplifies the underestimation of the viscosity in the next time step. As a result, flow velocities change with changing time step size. Avalanche codes such as RAMMS (Christen et al., 2007) deal with this problem by calibrating the model to data from previous events at the same location and similar conditions. But changes in release volume or position can lead to different accelerations and corresponding changes in the automatic time step control can alter the development of rheology over time. As long as a flow stage is reached where the flow stops accelerating, the influence on the final front velocity should be negligible. However, debrisflow models which do not iteratively adjust viscosity cannot accurately simulate impacts. Here, our model constitutes a significant improvement, since in the three-dimensional solver we presented, the viscosity bias was reduced by implementing a corrector step: taking the average between the viscosity at the beginning of the time step and the viscosity that corresponds to the velocity field at the end of the time step, the time step is solved again, leading to a better calculation of the velocity. This step can be repeated, according to user specifications, to correct the viscosity several times. Although this procedure increases numerical calculation time, it clearly reduces the time-step dependency of the simulation. Some dependency on the time step is still present when modeling the collapse of material columns, but the origin of this problem is different because it occurs also for Newtonian fluids.

\subsection{Effect of grid resolution on rheology}

Since the shear rate influences both viscosity models, viscosity is sensitive to grid resolution because the shear rate is averaged over the cell size. For flows over rough topography this may be less critical, but for laboratory flume experiments with thin shear bands the results may depend on grid resolution. When simulating laboratory flume experiments where debris-flow material accelerated in a relatively narrow and 


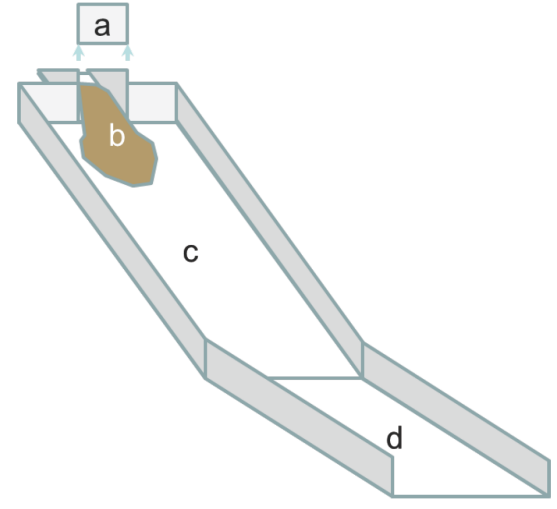

Figure 5. Iso-view sketch of the hillslope debris-flow flume. Material (b) is released from the reservoir at the top by the sudden vertical removal of a gate (a) and flows down a steep slope (c) followed by a gently inclined run-out plane (d).

short channel (Scheidl et al., 2013), a cell height of $1.5 \mathrm{~mm}$, which is of the order of the laboratory rheometer gap, was still not fine enough to reach the limit of grid sensitivity. The free model parameter $\tau_{00}$ influences the shear-rate-dependent term of the viscoplastic rheology model and can be used to adjust the simulation to the grid resolution. As long as the gravel phase and grid resolution do not change, it should be possible to model different water and clay contents based on one calibration test. However, as the composition changes, both $\tau_{y}$ and $\tau_{00}$ must change commensurately, since a change in yield stress affects the shear rate. Our procedure for adjusting to different mixtures based on one calibrated test is to perform one iteration step for the yield stress of the new mixture. By calculating $\tau_{y}$ based on the original $\tau_{00}$ value from the calibration test but with the new material composition, an updated yield stress of the new mixture is determined. Raising or lowering $\tau_{00}$ by the same ratio as the change from the original yield stress of the calibration test to the updated yield stress generates the final $\tau_{y}$ as it is applied to the simulation of the new mixture.

The viscosity of the granular phase is averaged over the cell faces to avoid discontinuous viscosity jumps between cells, which may cause instability due to the sensitivity of incompressible solvers to pressure-dependent viscosity. However, thin cells that allow a precise calculation of the shear gradient lead to a preferred direction of the smoothing of the granular phase's viscosity which may introduce physically unrealistic behavior. Cell length (in the flow direction), cell width, and cell height should at least be of the same order. Especially when front fingering is of interest, a grid resolution test should be carried out, ensuring that front instability is not caused by a large aspect ratio of the cell dimensions.

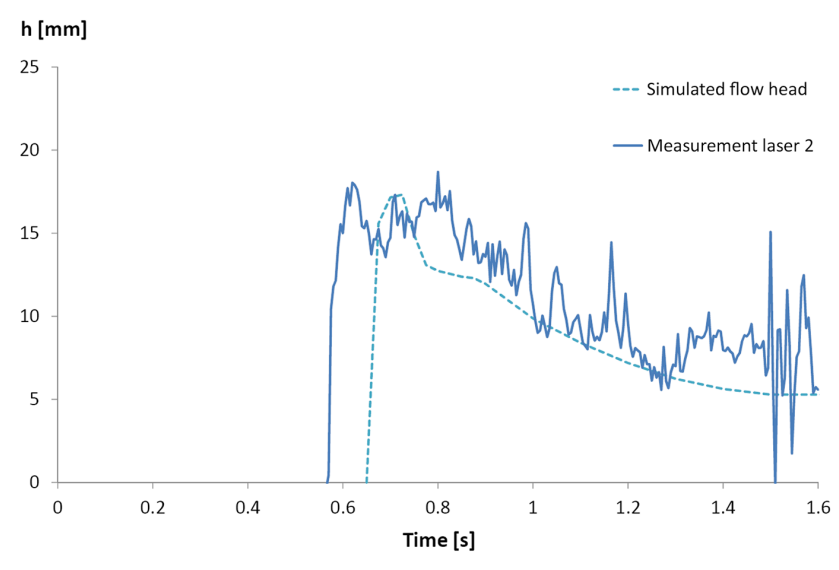

Figure 6. Laser measurement and corresponding simulated values of the flow head over time, $1 \mathrm{~m}$ downslope of the gate for hillslope debris-flow flume experiments with water content of $28.5 \%$. The laser data were box averaged over $10 \mathrm{~ms}$.

\section{Illustrative simulations}

Because the purpose of this paper is to illustrate the solver structure and model basis, we defer a detailed discussion of model performance to our next paper, in which the model is validated against laboratory tests, large-scale experiments, and natural hillslope debris-flow events. Here, we discuss only the efficiency of the solver itself, together with tests of the model accuracy in a gravity-driven open channel flow.

\subsection{Test case of a dam-break-released debris-flow mixture stopping on an inclined plane}

We chose an experiment from Hürlimann et al. (2015) as an illustration of model performance in the case of a flow stopping on an inclined plane without sidewall effects. The debris-flow experiment was carried out by releasing $0.01 \mathrm{~m}^{3}$ of debris-flow material from a $0.4 \mathrm{~m}$ wide reservoir into a $4.4 \mathrm{~m}$ long and $2 \mathrm{~m}$ wide, $30^{\circ}$ inclined plane followed by a $2.5 \mathrm{~m}$ long, $2 \mathrm{~m}$ wide, and $10^{\circ}$ inclined run-out section (Fig. 5). The flume was covered by a rubber layer with a burling consisting of flat circular discs of $4 \mathrm{~mm}$ diameter and about $0.3 \mathrm{~mm}$ height every $5 \mathrm{~mm}$ to increase roughness. The experimental sediment mixture used for model validation had $28.5 \%$ water content by weight and contained about $1.6 \%$ smectite, $8.8 \%$ other clay minerals, $27.8 \%$ silt, $47.8 \%$ sand, and $14 \%$ gravel. The corresponding bulk density was $1802 \mathrm{~kg} \mathrm{~m}^{-3}$. We determined the gravel friction angle $\delta=36^{\circ}$ as the angle of repose of the gravel mixture. To determine the angle of repose, we used a simple adaptation of the method of Deganutti et al. (2011), tilting a large box with loose material until a second failure of the material body occurred. The model parameter $\tau_{00}=41.33 \mathrm{~Pa}$ was calibrated to fit the observed run-out length. Laser-measured flow depths were available in the center of the flume, one meter downslope of the gate. Comparisons between measured 


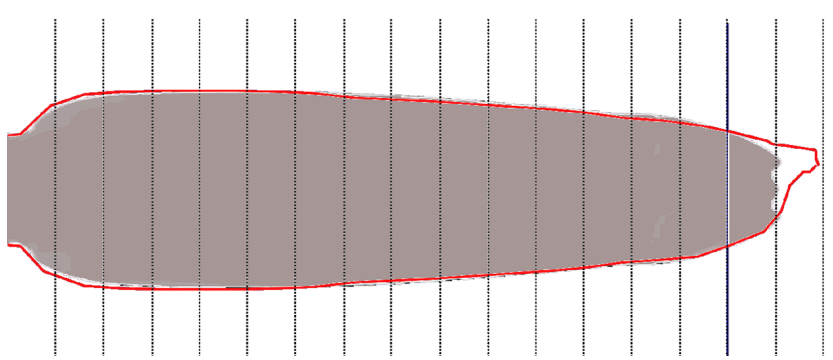

Figure 7. Simulated deposit (view from top in a hillslope debrisflow flume experiment) for a mixture with $28.5 \%$ water content and $\delta=36^{\circ}$. The thick solid lines indicate the experimental deposit and vertical thin lines represent the $20 \mathrm{~cm}$ spacing marks in the experimental slope.

and simulated flow depths at such small scales are only approximate due to the surface disturbance by coarser grains that cause significant fluctuations in surface elevation. However, the arrival time, the maximal flow depth, and the decay of surface elevation over time were considered to be suitable for comparison to the model. The model performance was evaluated by comparing the deposition patterns, travel times, and time series of flow depths in the simulations and experiments. The simulated flow depths reproduced the laser signal with respect to both time and amplitude (Fig. 6) and the predicted run-out deposit was in almost perfect agreement with the shape of the experimental deposit (Fig. 7). The simulation setup of the test case is included in the Supplement.

\subsection{Comparison to a drag-force-based Eulerian multiphase model}

In comparison to drag-force-based Eulerian multiphase models, the volume-of-fluid approach applied here significantly reduces calculation time. For an estimate, we compared our model with the OpenFOAM standard solver multiphaseEulerFoam. We selected the official tutorial case damBreak4phaseFine but turned the water phase into mercury to gain a three-phase test case, and applied the standard solver settings from the case to our model. On a CentOS 6.3 Linux machine with $31 \mathrm{GiB}$ memory and 16 Intel Xeon CPU E5-2665 @ 2.40 GHz processors, our model resulted in a 5.5 times faster calculation with a comparable collapse of material columns (Fig. 8). For the sake of completeness, our calculation included one iterative viscosity correction step, thus the model efficiency can be estimated to be about 10 times higher than a drag-force-based phase coupling approach.
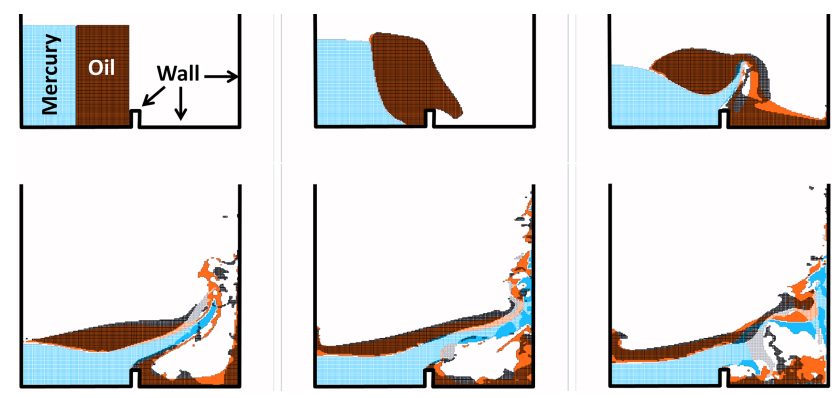

Figure 8. Phase positions in a dam break standard test-case simulation using a drag-based three-phase multiphaseEulerFoam simulation (air is transparent) as background shapes with the corresponding phase positions of our model as wire frame in front (with white mercury and black oil). The visualized time steps correspond to 0 , $0.1,0.2,0.3,0.4$, and $0.5 \mathrm{~s}$.

\section{Conclusions}

The new debris-flow solver has two main strengths. First, it can model three-dimensional flows and their impact against complexly shaped objects, representing the processes at a high level of detail and reasonable computational costs. Second, its design allows simulating different debris-flow material compositions with a reduced calibration process as long as the simulation grid does not change, because the calibration parameters $\tau_{00}$ and $n$ are largely insensitive to changes in water content, channel roughness, or release volume. Due to the pressure- and shear-dependent rheology used here, realistic deposit geometries and release dynamics can be achieved, as presented and discussed on the basis of test cases in the next paper. By systematically excluding unknown parameters from the model architecture and by accounting for known flow phenomena in a simplified way, we have developed a debris-flow model whose parameters can be roughly estimated from material composition, leaving only two calibration parameters. The concept is promising; however, important parts of phase interactions are neglected in favor of lower numerical costs.

\section{Code availability}

The source code can be downloaded from the supplementary application.zip. Please follow the instructions given in the README.pdf file for installation. 


\section{Appendix A}

The following section describes the detailed implementation of the PISO iteration procedure as described in Deshpande et al. (2012). By applying the continuum surface force model of Brackbill et al. (1992), the volume integral of Eq. (14) is given as

$$
\begin{aligned}
& \int_{\Omega_{i}} \frac{\partial \rho \boldsymbol{U}}{\partial t} \mathrm{~d} V+\int_{\partial \Omega_{i}}(\rho \boldsymbol{U} \boldsymbol{U}) \cdot \boldsymbol{n} \mathrm{d} S \\
& \quad=-\int_{\Omega_{i}} \nabla p_{d} \mathrm{~d} V-\int_{\Omega_{i}} \boldsymbol{g} \cdot \boldsymbol{x} \nabla \rho \mathrm{d} V+\int_{\Omega_{i}} \sigma \kappa \nabla \alpha_{1} \mathrm{~d} V \\
& \quad+\int_{\partial \Omega_{i}}(\mu \nabla \boldsymbol{U}) \cdot \boldsymbol{n} \mathrm{d} S+\int_{\Omega_{i}} \nabla \boldsymbol{U} \cdot \nabla \mu \mathrm{d} V .
\end{aligned}
$$

The computational domain is discretized into finitevolume cells. Each cell is considered as the owner of exactly one face that it shares with an adjacent neighbor cell, thus each face has a defined owner cell. A surface normal vector $\boldsymbol{S}_{f}$ with magnitude equal to the surface area of the face is defined on the face pointing outward from the owner cell (Fig. A1). The value at face $f$ of any variable $\chi$ calculated in the cell centers as $\chi_{P}$ and $\chi_{N}$ (Fig. A1) can be derived by interpolation using a mixture of central and upwind schemes:

$\chi_{f}=\gamma\left(\chi_{P}-\chi_{N}\right)+\chi_{N}$,

with a weighting factor $\gamma$ that can account for the flow direction based on the chosen interpolation scheme and flux limiter. In case of a linear interpolation scheme and a flux limiter $\psi, \gamma$ can be defined as

$\gamma=\psi \frac{f N}{d}+(1-\psi) \frac{\phi_{f}}{\left|\phi_{f}\right|}$,

where $d$ is the distance between the cell centers $P$ and $N$ and $f N$ is the distance from the face center to the cell center $N$. The face flux denoted as $\phi_{f}$ serves as a switch to account for the flow direction since it turns negative when the flow is from $N$ to $P$ (Berberović et al., 2009). Several limiters are implemented (OpenFOAM-Foundation, 2016b); we chose the van Leer scheme and assumed uniform grid spacing to simplify the following explanations with $f N / d=0.5$.

Variables that are evaluated at the cell faces are subscripted by $f$. Due to stability problems that arise from the pressure-velocity coupling in collocated meshes (Ferziger and Peric, 2002), the pressure is solved for the cell centers, whereas the velocity is interpolated to the cell faces within the PISO loop.

With the switch function

$\zeta\left(\phi_{f}\right)=\frac{\phi_{f}}{\left|\phi_{f}\right|}$, the velocity $\boldsymbol{U}_{f}$ at face $f$ can be written based on Eqs. (A2) and (A3) as

$\boldsymbol{U}_{f}=\frac{\boldsymbol{U}_{P}}{2}\left(1+\zeta\left(\phi_{f}\right)(1-\psi)\right)+\frac{\boldsymbol{U}_{N}}{2}\left(1-\zeta\left(\phi_{f}\right)(1-\psi)\right)$,

and the corresponding face-perpendicular velocity gradient is given by Deshpande et al. (2012) as

$\nabla_{f}^{\perp} \boldsymbol{U}=\frac{\boldsymbol{U}_{\boldsymbol{N}}-\boldsymbol{U}_{\boldsymbol{P}}}{|d|}$.

At the present time step $t^{n}$, the phase-averaged density of the next time step $\rho^{n+1}$ is known from solving the transport equations. In a first approximation, the corresponding viscosity field $\mu^{n+1}$ can be derived accordingly. A simplified formulation of the momentum Eq. (A1) without pressure, surface tension, and gravity terms discretized for cell $P$ could then be formulated as

$$
\begin{aligned}
& \frac{\left(\rho^{n+1} \widetilde{\boldsymbol{U}}\right)-\left(\rho^{n} \boldsymbol{U}^{n}\right)}{\Delta t}\left|\Omega_{P}\right|+\sum_{f \in \partial \Omega_{i}} \rho^{n}{ }_{f} \phi^{n}{ }_{f} \widetilde{\boldsymbol{U}}_{f} \\
& =\sum_{f \in \partial \Omega_{i}} \mu^{n+1}{ }_{f} \nabla^{\perp}{ }_{f} \widetilde{\boldsymbol{U}}\left|\boldsymbol{S}_{f}\right|+\nabla \boldsymbol{U}^{n} \cdot \nabla \mu^{n+1}\left|\Omega_{P}\right| .
\end{aligned}
$$

The tilde stands for the velocity at cell $P$ predicted in the current iterative step, for which Eq. (A7) yields an explicit expression. The sum over the face density fluxes on the left hand side of Eq. (A7) are known from the mass flux $\phi_{\rho}$ derived from Eq. (9).

For that purpose, Eqs. (A5) and (A6) are inserted into Eq. (A7) using the velocity of the prior iteration step, $\boldsymbol{U}^{m}$, in all neighbor cells (Deshpande et al., 2012). The explicit expression for the estimated velocity is

$A_{P} \widetilde{\boldsymbol{U}}=H\left(\boldsymbol{U}^{m}\right)$,

and by including surface tension and gravity this leads to

$\widetilde{\boldsymbol{U}}=\frac{H\left(\boldsymbol{U}^{m}\right)}{A_{P}}+\frac{\sigma \kappa \nabla \alpha_{1}^{n+1}}{A_{P}}-\frac{\boldsymbol{g} \cdot \boldsymbol{x} \nabla \rho}{A_{P}}$.

The detailed composition of $H\left(\boldsymbol{U}^{m}\right)$ and $A_{P}$ formulated with respect to the splitting between neighbor and owner cells can be found in Deshpande et al. (2012); here it is sufficient to keep in mind that $H\left(\boldsymbol{U}^{m}\right)$ contains all off-diagonal contributions of the linear system.

The next step is to assemble the approximated face flux 


$$
\begin{aligned}
\widetilde{\phi_{f}} & =\left(\frac{H\left(\boldsymbol{U}^{m}\right)}{A_{P}}\right)_{f} \cdot \boldsymbol{S}_{f}+\left(\frac{(\sigma \kappa)^{n+1}\left(\nabla^{\perp} \alpha_{1}\right)^{n+1}}{A_{P}}\right)_{f}\left|\boldsymbol{S}_{f}\right| \\
& -\left(\frac{(\boldsymbol{g} \cdot \boldsymbol{x})^{n+1}\left(\nabla^{\perp} f \rho\right)^{n+1}}{A_{P}}\right)_{f}\left|\boldsymbol{S}_{f}\right|,
\end{aligned}
$$

where the subscript $f$ indicates that the variable values at the faces are used. The final flux is found by adding the pressure contribution

$\phi_{f}^{m+1}=\widetilde{\phi_{f}}-\left(\frac{\nabla_{f}^{\perp} p_{d}^{m+1}}{A_{P}}\right)_{f}\left|\boldsymbol{S}_{f}\right|$.

The sum of the flux over the cell faces needs to be zero due to mass conservation for the incompressible flow

$\sum_{f \in \partial \Omega_{i}} \phi_{f}^{m+1}=0$.

Thus, the pressure is defined by the linear equation system for the updated pressure $p_{d}^{m+1}$

$$
\sum_{f \in \partial \Omega_{i}}\left(\frac{\nabla^{\perp} p_{d}^{m+1}}{A_{P}}\right)_{f}\left|\boldsymbol{S}_{f}\right|=\sum_{f \in \partial \Omega_{i}} \widetilde{\phi_{f}},
$$

and can be solved with the preconditioned conjugate gradient (PCG) algorithm, to mention one of several options implemented in OpenFOAM. With the updated pressure $p_{d}{ }^{m+1}$, the face fluxes $\phi^{m+1}$ are derived from Eq. (A11) and the updated velocity field $\boldsymbol{U}^{m+1}$ is obtained from the explicit velocity correction

$$
\begin{aligned}
\boldsymbol{U}^{m+1}= & \widetilde{\boldsymbol{U}}+\left(\frac{1}{A_{P}}\right)\left(\sum_{f \in \partial \Omega_{i}} \frac{\left(\boldsymbol{S}_{f} \otimes \boldsymbol{S}_{f}\right)}{\left|\boldsymbol{S}_{f}\right|}\right)^{-1} \\
& \cdot\left(\sum_{f \in \partial \Omega_{i}}\left(\frac{\phi^{m+1}{ }_{f}-\widetilde{\boldsymbol{U}}_{f} \cdot \boldsymbol{S}_{f}}{\left(\frac{1}{A_{P}}\right)_{f}}\right) \frac{\boldsymbol{S}_{f}}{\left|\boldsymbol{S}_{f}\right|}\right)
\end{aligned}
$$

which is the end of the PISO loop. After updating the index $m$ to $m+1$, the iteration restarts by recalculating $H$ with the updated velocity from Eq. (A8), repeating the loop until a divergence-free velocity field is found.

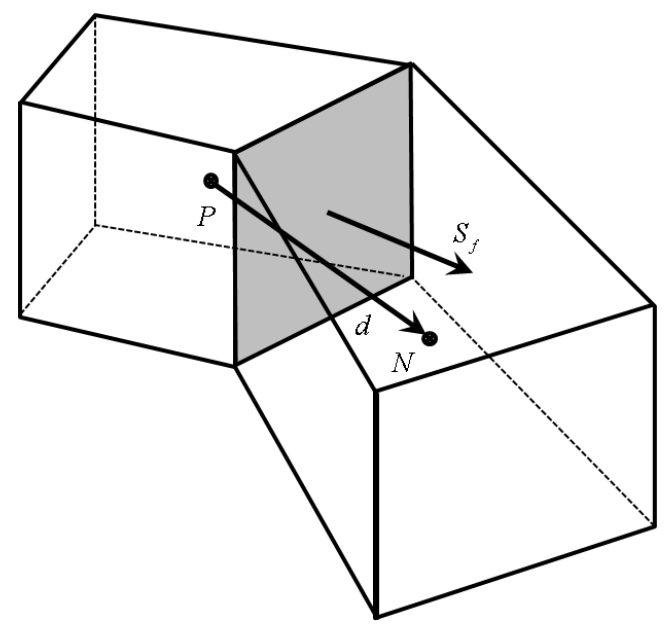

Figure A1. Sketch of two adjacent cells $P$ and $N$ and the shared face $f$ owned by cell $P . S_{f}$ is the face surface normal vector while $d$ denotes the distance vector from cell center $P$ to cell center $N$. 


\section{The Supplement related to this article is available online at doi:10.5194/gmd-9-2909-2016-supplement.}

Acknowledgements. We thank Shiva Pudasaini, Johannes Hübl, and Eugenio Oñate for thoughtful critiques and suggestions.

Edited by: J. Neal

Reviewed by: R. M. Iverson and two anonymous referees

\section{References}

Ancey, C.: Plasticity and geophysical flows: a review, J. NonNewton. Fluid, 142, 4-35, 2007.

Ancey, C. and Jorrot, H.: Yield stress for particle suspensions within a clay dispersion, J. Rheol., 45, 297-319, 2001.

Balmforth, N. and Frigaard, I.: Viscoplastic fluids: from theory to application, J. Non-Newton. Fluid, 142, 1-3, 2007.

Berberović, E., van Hinsberg, N. P., Jakirlić, S., Roisman, I. V., and Tropea, C.: Drop impact onto a liquid layer of finite thickness: Dynamics of the cavity evolution, Phys. Rev. E, 79, 036306, doi:10.1103/PhysRevE.79.036306, 2009.

Berger, C., McArdell, B. W., and Schlunegger, F.: Direct measurement of channel erosion by debris flows, Illgraben, Switzerland, J. Geophys. Res.-Earth, 116, F01002, doi:10.1029/2010JF001722, 2011.

Bouchut, F., Fernandez-Nieto, E. D., Mangeney, A., and NarbonaReina, G.: A two-phase shallow debris flow model with energy balance, ESAIM-Math. Model Num., 49, 101-140, 2015.

Bozhinskiy, A. N. and Nazarov, A. N.: Two-phase model of debris flow, in: 2nd International Conference on Debris-Flow Hazards Mitigation, pp. 16-18, Teipei, Taiwan, 2000.

Brackbill, J. U., Kothe, D. B., and Zemach, C.: A continuum method for modeling surface tension, J. Comput. Phys., 100, 335-354, 1992.

Brodani-Minussi, R. and deFreitas Maciel, G.: Numerical Experimental Comparison of Dam Break Flows with non-Newtonian Fluids, J. Braz. Soc. Mech. Sci., 34-2, 167-178, 2012.

Bugnion, L., McArdell, B. W., Bartelt, P., and Wendeler, C.: Measurements of hillslope debris flow impact pressure on obstacles, Landslides, 9, 179-187, 2012.

Christen, M., Bartelt, P., and Gruber, U.: RAMMS - a Modelling System for Snow Avalanches, Debris Flows and Rockfalls based on IDL, Photogramm. Fernerkun., 4, 289-292, 2007.

Coussot, P., Laigle, D., Aratano, M., Deganuttil, A., and Marchi, L.: Direct determination of rheological characteristics of debris flow, J. Hydraul. Eng.-ASCE, 124, 865-868, 1998.

Deganutti, A., Tecca, P., and Genevois, R.: Characterization of friction angles for stability and deposition of granular material, in: Italian Journal of Engineering and Environment: 5th International Conference on Debris-Flow Hazards: Mitigation, Mechanics, Prediction and Assessment, 313-318, Padua, Italy, 2011.

Deshpande, S. S., Anumolu, L., and Trujillo, M. F.: Evaluating the perfomance of the two-phase flow solver interFoam, Computational Science and Discovery, 5, 1-33, 2012.
Domnik, B. and Pudasaini, S.: Full two-dimensional rapid chute flows of simple viscoplastic granular materials with a pressuredependent dynamic slip-velocity and their numerical simulations, J. Non-Newton. Fluid, 173-174, 72-86, 2012.

Domnik, B., Pudasaini, S., Katzenbach, R., and Miller, S.: Coupling of full two-dimensional and depth-averaged models for granular flows, J. Non-Newton. Fluid, 201, 56-68, 2013.

Ferziger, J. H. and Peric, M.: Computational Methods for Fluid Dynamics, 3rd Edn., Springer, Berlin, Germany, 2002.

Fischer, H. B.: Longitudinal Dispersion in Laboratory and Natural Streams, PhD thesis, California Institute of Technology, 1966.

Forterre, Y. and Pouliquen, O.: Flows of dense granular media, Annu. Rev. Fluid Mech., 40, 1-24, 2008.

Guthrie, R., Mitchell, J., Lanquaye-Opoku, N., and Evans, S.: Extreme weather and landslide initiation in coastal British Columbia, J. Eng. Geol. Hydrogeol., 43, 417-428, 2010.

Hänsch, S., Lucas, D., Höhne, T., Krepper, E., and Montoya, G.: Comparative simulations of free surface flows using VOFmethods and a new approach for multi-scale interfacial structures, in: Proceedings of the ASME 2013 Fluids Engineering Summer Meeting, Incline Village, Nevada, USA, 2013.

Hilker, N., Badoux, A., and Hegg, C.: The Swiss flood and landslide damage database 1972-2007, Nat. Hazards Earth Syst. Sci., 9, 913-925, doi:10.5194/nhess-9-913-2009, 2009.

Hill, D.: The Computer Simulation of Dispersed Two-Phase Flows, $\mathrm{PhD}$ thesis, Imperial College, University of London, 1998.

Hirt, B. and Nichols, B.: Volume of Fluid (VOF) Method for the Dynamics of Free Boundaries, J. Comput. Phys., 39, 201-225, 1981.

Hoang, D., van Steijn, V., Kreutzer, M., and Kleijn, C.: Modeling of Low-Capillary Number Segmented Flows in Microchannels Using OpenFOAM, in: Numerical Analysis and Applied Mathematics ICNAAM 2012, AIP Conf. Proc., Vol. 1479, 86-89, Kos Island, Greece, 2012.

Hürlimann, M., McArdell, W., and Rickli, C.: Field and laboratory analysis of the runout characteristics of hillslope debris flows in Switzerland, Geomorphology, 232, 20-32, doi:10.1016/j.geomorph.2014.11.030, 2015.

Imran, J., Parker, G., Locat, J., and Lee, H.: 1D numerical model of muddy subaqueous and subaerial debris flows, J. Hydraul. Eng.ASCE, 127, 959-968, 2001.

Ishii, M.: Thermo-Fluid Dynamic Theory of Two-Phase Flow, Eyrolles, Paris, 1975.

Issa, R.: Solution of the implicitly discretized fluid-flow equations by operator-splitting, J. Comput. Phys., 62, 40-65, 1986.

Iverson, R. and Denlinger, P.: Flow of variably fluidized granular masses across three-dimensional terrain: 1 . Coulomb mixture theory, J. Geophys. Res., 106, 537-552, 2001.

Jop, P., Forterre, Y., and Pouliquen, O.: A constitutive law for dense granular flows, Nature, 441, 727-730, 2006.

Leonardi, A., Wittel, F. K., Mendoza, M., Vetter R., and Hermann, H. J.: Particle-Fluid-Structure Interaction for Debris Flow Impact on Flexible Barriers, Comput.-Aided Civil Infrastruct. Eng., 31, 323-333, 2016.

OpenFOAM-Foundation: OpenFOAM Standard Solvers, Website User Guide of OpenFOAM, available at: http://www.openfoam.org/docs/user/standard-solvers.php, last access: 12 January 2016 . 
OpenFOAM-Foundation: OpenFOAM Standard Schemes, Website User Guide of OpenFOAM, available at: http://cfd. direct/openfoam/user-guide/fvSchemes/, last access: 12 December $2016 b$.

Petley, D. N., Hearn, G. J., Hart, A., Rosser, N. J., Dunning, S. A., Oven, K., and Mitchell, W. A.: Trends in landslide occurence in Nepal, Nat. Hazards, 43, 23-44, 2007.

Pitman, E. and Le, L.: A two-fluid model for avalanche and debris flows, Philos. T. R. Soc. A, 363, 1573-1602, 2005.

Pudasaini, S.: A general two-phase debris flow model, J. Geophys. Res., 117, F03010, doi:10.1029/2011JF002186, 2012.

Pudasaini, S. P., Wang, Y., and Hutter, K.: Modelling debris flows down general channels, Nat. Hazards Earth Syst. Sci., 5, 799819, doi:10.5194/nhess-5-799-2005, 2005a.

Pudasaini, S., Hsiau, S. S., Wang, Y., and Hutter, K.: Velocity measurements in dry granular avalanches using particle image velocimetry technique and comparison with theoretical predictions, Phys. Fluids, 17, 093301, doi:10.1063/1.2007487, 2005b.

Pudasaini, S., Hutter, K., Hsiau, S. S., Tai, S. C., Wang, Y., and Katzenbach R.: Rapid flow of dry granular materials down inclined chutes impinging on rigid walls, Phys. Fluids, 19, 053302, doi:10.1063/1.2726885, 2007.

Remaitre, A., Malet, J., Maquaire, O., Ancey, C., and Locat, J.: Flow behaviour and runout modelling of a complex derbis flow in a clay-shale basin, Earth Surf. Proc. Land., 30, 479-488, 2005.

Renardy, M.: Some remarks on the Navier-Stokes Equations with a pressure-dependent viscosity, Commun. Part. Diff. Eq., 11, 779$793,1986$.
Schatzmann, M., Fischer, P., and Bezzola, G. R.: Rheological Behavior of Fine and Large Particle Suspensions, J. Hydraul. Eng.ASCE, 796, 391-430, 2003.

Scheidl, C., Chiari, M., Kaitna, R., Müllegger, M., Krawtschuk, A., Zimmermann, T., and Proske, D.: Analysing Debris-Flow Impact Models, Based on a Small Scale Modelling Approach, Surv. Geophys, 34, 121-140, 2013.

Tang, C., Zhu, J., Ding, J., Cui, X. F., Chen, L., and Zhang, J. S.: Catastrophic debris flows triggered by a 14 August 2010 rainfall at the epicenter of the Wenchuan earthquake, Landslides, 8, 485497, doi:10.1007/s10346-011-0269-5, 2011.

von Boetticher, A.: Flexible Hangmurenbarrieren: Eine numerische Modellierung des Tragwerks, der Hangmure und der Fluid-Struktur-Interaktion, $\mathrm{PhD}$ thesis, Technische Universität München, 2013.

von Boetticher, A., Turowski, J. M., McArdell, B. W., Rickenmann, D., Hürlimann, M., Scheidl, C., and Kirchner, J. W. DebrisInterMixing-2.3: a Finite Volume solver for three dimensional debris flow simulations based on a single calibration parameter - Part 2: Model validation, Geosci. Model Dev. Discuss., 8, 6379-6415, doi:10.5194/gmdd-8-6379-2015, 2015.

Yu, B., Ma, Y., and Qi, X.: Experimental Study on the Influence of Clay Minerals on the Yield Stress of Debris Flows, J. Hydraul. Eng.-ASCE, 139, 364-373, 2013. 